\title{
Variability in the measurement of the methylation status of lung cancer-related genes in bronchial secretions
}

\author{
LAURA MILLARES $^{1-3}$, ANTONI ROSELL ${ }^{2,4}$, LAIA SETÓ ${ }^{1,2,4-6}$, JOSÉ SANZ ${ }^{2,5}$, \\ FELIPE ANDREO $^{2,5}$ and EDUARD MONSÓ ${ }^{2,3,6}$ \\ ${ }^{1}$ Fundació Parc Taulí, Sabadell/Badalona; ${ }^{2}$ CIBER de Enfermedades Respiratorias, CIBERES, Bunyola; \\ ${ }^{3}$ Universitat Autònoma de Barcelona, Esfera UAB, Barcelona; ${ }^{4}$ Department of Respiratory Medicine, \\ Hospital Universitari Bellvitge-IDIBELL, Barcelona; ${ }^{5}$ Department of Respiratory Medicine, \\ Hospital Universitari Germans Trias i Pujol, Badalona; ${ }^{6}$ Department of Respiratory Medicine, \\ Hospital Universitari Parc Taulí, Sabadell, Spain
}

Received May 6, 2014; Accepted June 12, 2014

DOI: $10.3892 /$ or.2014.3364

\begin{abstract}
Assessment of the methylation status of genes related to the development of lung cancer (LC) in bronchial secretions has been proposed as a biomarker for early detection. Several techniques are available to detect gene methylation, and the method chosen may have an effect on the results. A cross-sectional study was conducted in which the methylation status of DAPK, CDKN2A (p16) and RASSF1A genes in sputum and bronchial washing (BW) from subjects at risk for LC was analyzed. The methylation results of both samples were compared, considering BW as the reference. Results obtained by methylation-sensitive PCR (MSP) were validated by methylation-sensitive high-resolution melting (MS-HRM). The methylation results obtained in sputum and BW samples did not show statistically significant differences for any of the three genes analyzed in 65 subjects (McNemar test $>0.05$ ). Concordant results between sputum and BW were found in 40 patients for DAPK $(61 \%)$, in 52 patients for p16 (80\%) and in 63 patients for RASSF1 (97\%). More methylated samples were found in BW, however, and sputum sensitivities and specificities for the identification of methylation status were 44 and $72 \%$ for DAPK gene, 21 and $94 \%$ for p16 and 100 and 98\% for RASSF1A, respectively. When MSP results were validated by MS-HRM, DAPK and p16 gene samples methylated by MSP appeared to be unmethylated by MS-HRM. One sample showing methylation of RASSF1A gene also showed methylation when tested following MS-HRM procedure. Sputum and BW samples may be considered equally valid for the identification of methylated genes in bronchial secretions. The low sensitivity of sputum for the assessment of the methylation status of DAPK and p16 genes, however, suggests that the analysis of two or more sputum
\end{abstract}

Correspondence to: Dr Eduard Monsó, Department of Respiratory Medicine, Hospital Universitari Parc Taulí, Sabadell, Spain E-mail: emonso@tauli.cat

Key words: methylation-sensitive PCR, methylation-sensitive highresolution melting, bronchial washing, sputum samples, or of a BW obtained semi-invasively, would be needed to attain higher reliability, together with the use of confirmatory techniques for positive results.

\section{Introduction}

DNA methylation determines spatial and temporal silencing of gene expression (1), and the aberrant methylation of $\mathrm{CpG}$ islands located in the promoter regions of genes that regulate cell proliferation is a frequent event in most types of cancers (2). Methylation is often an early phenomenon during carcinogenesis (3-5), and the methylation status of specific genes has been proposed as an appropriated biomarker for early detection of lung cancer (LC) $(4,6,7)$. In patients at risk for this disease, the assessment of the methylation status of these genes in bronchial secretions, such as sputum, bronchial washing and bronchial lavage may be useful for the identification of subjects candidate for additional procedures (6). Most of the studies requiring respiratory samples have relied on sputum for the definition of biomarkers with predictive ability $(3,8)$. Sputum samples are easily obtained, but contain a mixture of inflammatory and oral cells, with an epithelial fraction that comprises $<3 \%$ of the sample. This cellular heterogeneity, which varies across subjects, limits the possibilities for methylation status assessment (3). Bronchial washing (BW) samples can be obtained semi-invasively and contain a higher proportion of epithelial cells (9). Therefore, BW may be more appropriate for methylation studies of bronchial cells.

Methods based on methylation-sensitive PCR (MSP) primers are used to identify low levels of DNA methylation and may be useful for bronchial secretions (10-12). With this procedure, two sets of primers allow the amplification of the sequence of interest, with one pair recognizing the methylated sequence and the other pair the unmethylated one. Subsequent product analysis is performed in most cases by gel electrophoresis (13). MSP does not require specialized equipment and is sufficiently sensitive to identify $0.1 \%$ of the methylated template. However, the method is non-quantitative and does not fully avoid false-positive results in single sample analyses, since it is not a closed tube method $(14,15)$. 
Methylation-sensitive high-resolution melting (MS-HRM) analysis is a recently developed methodology with enormous potential for the detection of DNA sequence changes (16). With this technique, sequence differences between methylated and unmethylated DNA obtained after bisulfite treatment can be analyzed by melting curve analysis. As the PCR product originating from the methylated allele has a different GC content from the PCR product obtained from the unmethylated variant, the two products have distinct melting temperatures (17). In this case, the methylation level is estimated by comparing the melting profiles of samples and standards of known ratios of methylated and unmethylated DNA (15).

The goal of this study was to determine whether methylation results in sputum samples parallel results obtained in $\mathrm{BW}$, a sample with a higher proportion of epithelial cells and the effect of the used methodology on these results. To do so we analyzed the methylation levels of three genes related to cancer development in sputum and BW obtained from subjects at risk for LC (7). Death-associated protein kinase (DAPK), involved in apoptosis; cyclin-dependent kinase inhibitor 2A gene (CDKN2A/p16), a tumor-suppressor gene which plays a key role in cell cycle control; and ras effector homolog 1 gene (RASSF1A), related to cell cycle progression at the G1-S transition, were the assessed genes. Sputum and BW were analyzed, and the methylation status after two different amplification methods, MSP and MS-HRM, were compared to assess the impact of the methodology on the identification of DNA methylation in these bronchial samples.

\section{Materials and methods}

Ethics statement. The research protocol was approved by the ethics committees of the affiliated hospitals, and written informed consent was obtained from all subjects. Sputum and BW recoveries were obtained in accordance with scheduled explorations for standard care, which included diagnostic and follow-up bronchoscopies for the enrolled individuals.

Design and population. This is a cross-sectional study in which bronchial secretions of subjects at risk for LC were analyzed to detect the methylation levels of three carcinogenesis-related genes. Included subjects were smokers of $>30$ pack-years with no signs or symptoms of LC and a normal chest X-ray when examined and not treated for any cancer in the previous 10 years.

Sampling procedure. Induced sputum samples were obtained and processed within $60 \mathrm{~min}$ at enrollment using standard methods $(18,19)$. Briefly, the subject was pretreated with an inhaled $\beta 2$-agonist $10 \mathrm{~min}$ before the nebulization of isotonic saline $(0.9 \%)$, followed by increasing concentrations of hypertonic saline (3,4 and 5\%) for 7 min with each concentration. After each induction, the patient attempted to provide a sputum sample by coughing, and the nebulization procedure was discontinued when the sputum volume collected was equal or higher than $1 \mathrm{ml}$. Sputum induction was followed by a bronchoscopy performed under local anesthesia and sedation, using a flexible video bronchoscope (BF180; Olympus Optical Co., Tokyo, Japan). Local anesthesia and sedation were achieved using topical lidocaine spray and intravenous midazolam respectively, in accordance with standard recommendations $(20,21)$. BW was obtained aspirating bronchial secretions through the working channel during the procedure.

Samples and DNA extraction. Sputum and BW samples were diluted with $5 \mathrm{ml}$ of a 1:10 dilution of dithiothreitol (Sputasol; Oxoid, Basingstoke, Hants, UK), centrifuged and the cellular fraction of the sample separated and stored at $-80^{\circ} \mathrm{C}$ until DNA extraction. The QIAamp DNA Mini Kit and the QIAcube (both from Qiagen, Hilden, Germany) were used for extraction, in accordance with the manufacturer's instructions, and DNA was quantified with a Nanodrop ND-1000 spectrophotometer (NanoDrop Technologies Inc., Wilmington, DE, USA). Universal methylated DNA and unmethylated DNA (Chemicon, Millipore, Billerica, MA, USA) were used as a fully methylated positive control and unmethylated negative control, respectively.

Bisulfite modification. One miligram of DNA was modified by treatment with sodium bisulfite and then purified with the Wizard DNA purification kit (Promega, Madison, WI, USA), desulfonated with $\mathrm{NaOH}$, precipitated with sodium acetate and ethanol, resuspended with $30 \mu \mathrm{l}$ of PCR grade $\mathrm{H}_{2} \mathrm{O}$ and stored at $-20^{\circ} \mathrm{C}$ for further determinations (22). For the MS-HRM study, DNA was modified using the EZ DNA Methylation-Gold kit (Zymo Research Co., Irvine, CA, USA) according to manufacturer's instructions.

Primer design. Previously described primers were used for MSP analysis $(22,23)$ (Table I). For HRM analysis, DAPK and RASSF1A primers were designed with Primer Express software (Applied Biosystems, Foster City, CA, USA), without any $\mathrm{CpG}$ dinucleotide in the sequence, determining the annealing temperature experimentally to avoid the PCR bias phenomenon. P16 primers for HRM analysis have been described elsewhere (24) (Table II).

Methylation-specific PCR (MSP). Two set of primers were used for specific amplification of each region of interest with this technique. One pair recognized the unmethylated sequence, whose cytosines were changed to uracils by bisulfite modification and the other pair the methylated sequence. Primer sequences and PCR conditions were adjusted for each pair of primers and are described in Table I. Positive and negative methylation controls were analyzed in each assay. PCRs were carried out in a volume of $20 \mu 1$, which contained $1 \mathrm{X}$ reaction buffer, $1.5 \mathrm{mM}$ of $\mathrm{MgCl}_{2}, 0.2 \mu \mathrm{M}$ of each primer, $1 \mathrm{mM}$ of dNTPs and 0.6 units of Taq polymerase (Qiagen). The procedure was performed on a Thermal Cycler 2720 (Applied Biosystems). After amplification, the products were visualized on $2 \%$ agarose gels with ethidium bromide staining (Fig. 1). Methylation assessment was conducting in duplicate, starting with bisulfite modification. A positive result in 1 out of 2 was taken as positive for methylation $(3,25)$.

Methylation-sensitive high-resolution melting (MS-HRM). PCR cycling and HRM analysis were performed on a LightCycler 480 II (Roche Applied Science, Mannheim, Germany). Reactions were carried out in a total volume of $10 \mu 1$ containing 2X PCR Master Mix, 2.5-3 $\mathrm{mM}$ of $\mathrm{MgCl}_{2}$, $2-3 \mu \mathrm{M}$ of each primer, depending on the gene studied and $2 \mu \mathrm{l}$ 
Table I. MSP primers.

\begin{tabular}{|c|c|c|c|c|}
\hline Gene & Sequence & $\begin{array}{c}\text { Annealing } \\
\text { temperature }\left({ }^{\circ} \mathrm{C}\right)\end{array}$ & $\begin{array}{l}\text { Amplicon } \\
\text { size (bp) }\end{array}$ & Spanned region \\
\hline p16 & $\begin{array}{l}\text { MF-ttattagagggtggggcggatcgcgtgc } \\
\text { MR-acccgaccccgaaccgcgaccgtaa } \\
\text { UF-ttattagagggtggggtggattgt } \\
\text { UR-caaccccaaaccacaaccataa }\end{array}$ & $\begin{array}{l}67 \\
65\end{array}$ & 154 & $\begin{array}{l}\text { ENSG000001478889 } \\
21,974,907- \\
21,974,753\end{array}$ \\
\hline DAPK & $\begin{array}{l}\text { MF-ggatagtcggatcgagttaacgtc } \\
\text { MR-ccctcccaaacgccga } \\
\text { UF-ggaggatagttggattgagttaatgtt } \\
\text { UR-caaatccctcccaaacaccaa }\end{array}$ & 60 & 108 & $\begin{array}{l}\text { ENSG00000196730 } \\
90,112,771- \\
90,112,879\end{array}$ \\
\hline RASSF1A & $\begin{array}{l}\text { MF-gggttttgcgagagcgcg } \\
\text { MR-gccaagcgcaaacaatcg } \\
\text { UF-ggttttgtgagagtgtgtttag } \\
\text { UR-cactaacaaacacaaaccaaac }\end{array}$ & $\begin{array}{l}62 \\
62\end{array}$ & 170 & $\begin{array}{l}\text { ENSG00000068028 } \\
50,378,432- \\
50,378,262\end{array}$ \\
\hline
\end{tabular}

MSP, methylation-sensitive PCR.

Table II. MS-HRM primers.

\begin{tabular}{|c|c|c|c|c|c|}
\hline Gene & Sequence & $\begin{array}{c}\text { Annealing } \\
\text { temperature }\left({ }^{\circ} \mathrm{C}\right)\end{array}$ & $\begin{array}{l}\text { Amplicon } \\
\text { size (bp) }\end{array}$ & $\begin{array}{l}\text { CpG between } \\
\text { primers }\end{array}$ & Spanned region \\
\hline p16 & $\begin{array}{l}\text { F-gaagaaagaggaggggttggttggttatt } \\
\text { R-acctactctccccetctccgcaa }\end{array}$ & 68 & 84 & 6 & $\begin{array}{l}\text { ENSG000001478889 } \\
21,974,931-847\end{array}$ \\
\hline DAPK & $\begin{array}{l}\text { F-gtttgtagggtttttattggt } \\
\text { R-actatcctcctcacactcc }\end{array}$ & 59 & 94 & 7 & $\begin{array}{l}\text { ENSG00000196730 } \\
90,112,712-806\end{array}$ \\
\hline RASSF1A & $\begin{array}{l}\text { F-gtttagtttggattttggg } \\
\text { R-aactcaataaactcaaactcc }\end{array}$ & 60 & 139 & 12 & $\begin{array}{l}\text { ENSG00000068028 } \\
50,378,338-199\end{array}$ \\
\hline
\end{tabular}

MS-HRM, methylation-sensitive high-resolution melting.

of the template. The annealing temperatures were experimentally determined for each assay to compensate for PCR bias, as shown in Table II.

Each assay was optimized so that no amplification was observed in the unmodified control or in the non-template control. Standard series of 100, 50, 30, 10 and 5\% methylation levels were prepared by diluting the fully methylated DNA into the unmethylated DNA and were used as controls. The methylation level of each sample was assessed by comparison of the PCR product melting profile and standards with a known ratio of methylated and unmethylated templates (Fig. 2). The PCR bias of each assay was successfully corrected with the annealing temperature and proportional amplifications of the standard with $50 \%$ of methylated DNA were seen in a background of unmethylated DNA. All samples were run in duplicate. Methylation assessment was conducting in duplicate, starting with bisulfite modification. A positive result in one out of two was taken as positive for methylation.

Statistical analysis. Data were analyzed using SPSS Statistical Software package version 18 (SPSS Inc., Chicago, IL, USA).
Results for categorical variables are expressed as absolute and relative frequencies and results for continuous variables as means and standard deviations (SD), or as medians and percentiles 25-75 (P25-P75) when the distribution was not normal.

First, clinical and functional variables of the studied subjects were described, and the methylation results obtained in sputum and in BW were compared. Considering BW as the reference, the sensitivity and specificity of sputum were calculated. Secondly, the results obtained after the two different PCR methods were compared, to determine their specific advantages in the study of bronchial secretions. All analyses were performed using the Chi-square, Fisher's exact, McNemar or Mann-Whitney U test as required. Statistical tests were two-sided, and a P-value of 0.05 or less was reported as statistically significant.

\section{Results}

Characteristics of the participants and analysis of respiratory secretions. Bronchial secretions from 65 subjects with an 


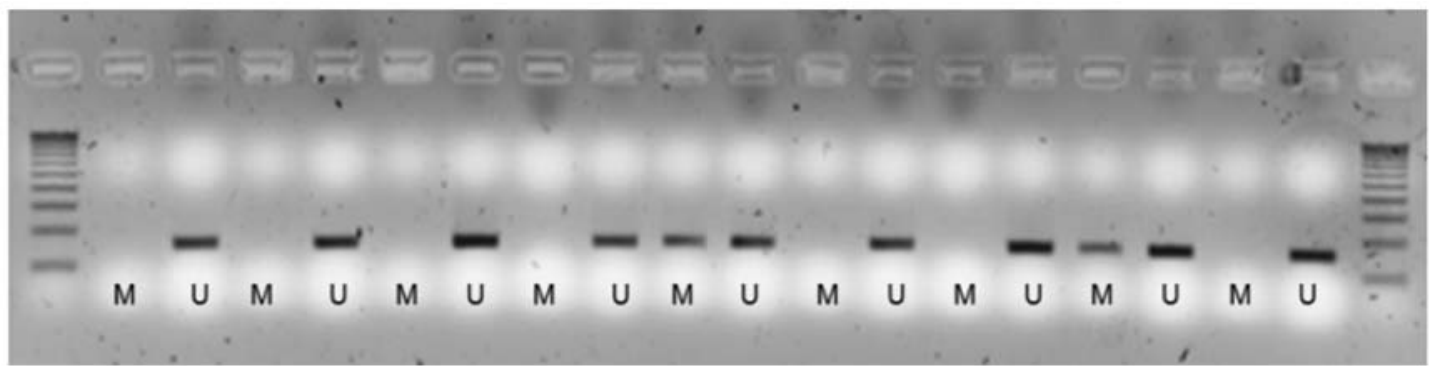

Figure 1. Agarose gel to detect the methylation status of the samples after MSP. MSP, methylation-sensitive PCR.
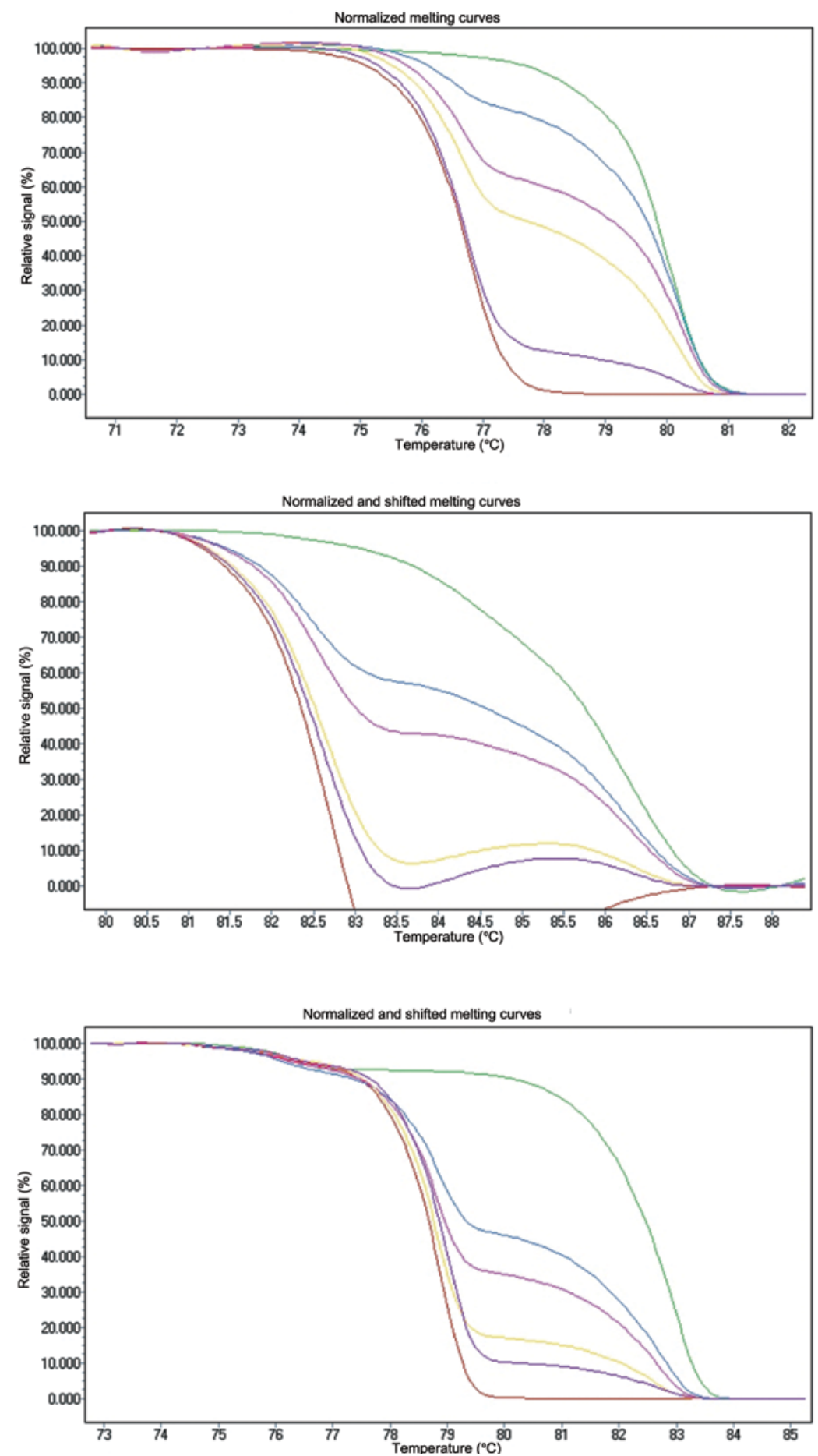

Figure 2. Normalized melting curves of the standards for the DAPK (A), p16 (B) and RASSF1A (C) genes. In green, 100\% methylated; in blue, 50\% methylated; in pink, 30\% methylated; in yellow, $10 \%$ methylated; in purple, $5 \%$ methylated and in red, unmethylated. 


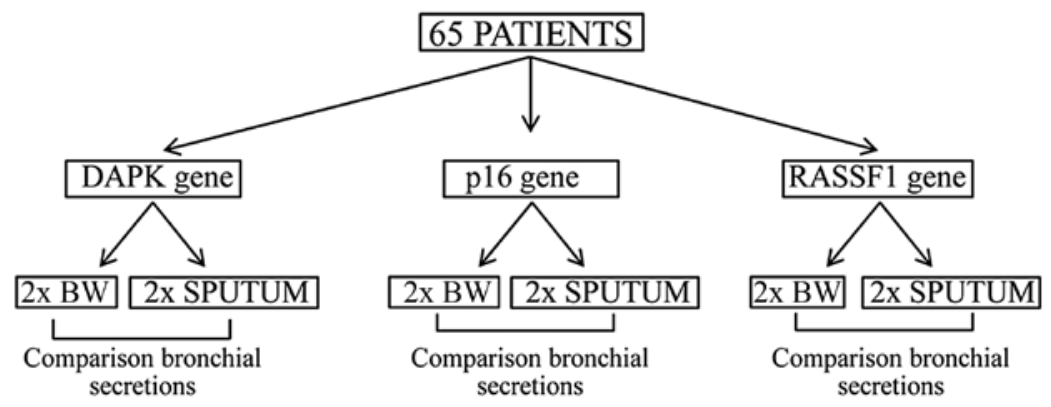

Figure 3. Flow chart showing the analysis comparing the methylation status in sputum and bronchial washing (BW).

average age of 62 years (SD 9.4) were analyzed in this study. Sixty-two of the enrolled subjects were men (95.4\%) and their lung function showed a mean post-bronchodilator forced expiratory volume in $1 \mathrm{sec}$ of $72 \%$ (SD 23) of the predicted value. All participants were current or former smokers with a heavy cumulative smoking history [median 48 (interquartile range 30-65) pack-years]. Ten subjects had a history of LC (15\%) but had been free from neoplastic disease the 10 previous years.

First, sputum and BW were manually modified and the methylation status of the DAPK, p16 and RASSF1A genes was determined by MSP (Fig. 3). Differences between these two samples were not statistically significant for any of the studied genes (DAPK, $\mathrm{P}=0.690$; $16, \mathrm{P}=0.092$; RASSF1, $\mathrm{P}=1.00$; McNemar test). Concordant results between sputum and BW were found in 40 patients for DAPK (61\%), in 52 patients for p16 (80\%) and in 63 patients for RASSF1 (97\%) (Fig. 4). More methylated samples were found in BW, however, and considering this sample as the reference, sputum sensitivity and specificity for the identification of methylation status were calculated. Sensitivities and specificities were 44 and $72 \%$ for the DAPK gene, 21 and $94 \%$ for p16 and 100 and $98 \%$ for RASSF1A, respectively.

Comparison of MSP and MS-HRM techniques. Forty samples of bronchial secretions were available for a second testing by MS-HRM, for the comparison of MSP and MS-HRM techniques. Twenty-three methylated samples for the DAPK gene and 6 for p16 after MSP emerged to be unmethylated with the MS-HRM technique, with a limit of detection of $5 \%$. For RASSF1A, the sample that appeared methylated by MSP showed the same result with MS-HRM.

\section{Discussion}

In the present study, although the methylation status of DAPK, p16 and RASSF1A genes was similar in single samples of sputum and BW, higher levels of positive results were obtained with BW for DAPK and p16 genes, a result attributable to the higher proportion of epithelial cells in this sample $(6,9)$. The lack of statistically significant differences between both samples of bronchial secretions confirms that sputum may be considered useful as a source of DNA for the identification of epigenetic changes in bronchial secretions, and the fact that this sample is obtained non-invasively offers a significant advantage for screening. Our results, however, suggest that single sputum samples may underestimate the methylation

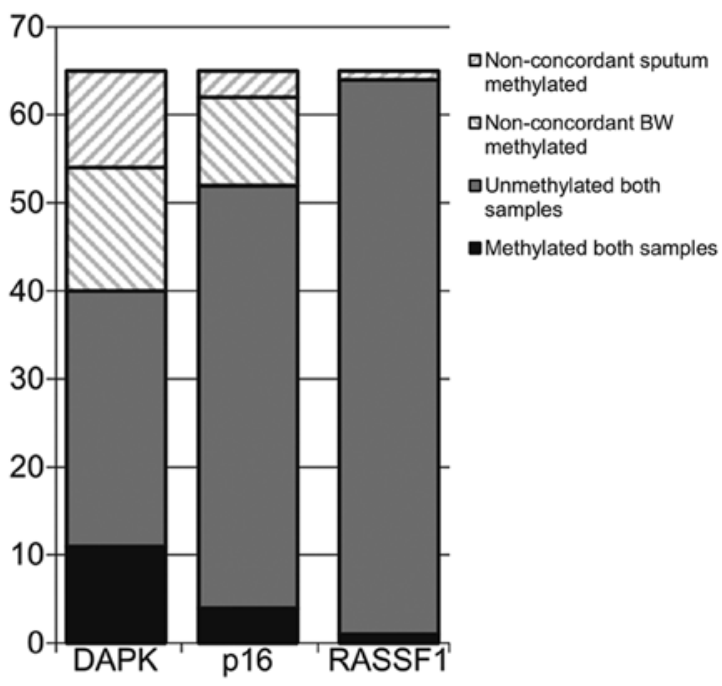

Figure 4. Results obtained after manual modification and MSP analysis of bronchial secretion samples. Methylation results in sputum and bronchial washings from 65 patients. MSP, methylation-sensitive PCR.

status of bronchial cells and support the use of repeated sputum samples, or its combination with BW, to guarantee an accurate assessment of the methylation status of the bronchial tree (26).

The methylation status was assessed again in a subgroup of samples using MS-HRM to compare the obtained results with two different techniques. This is a sensitive technique that analyzes DNA methylation in a semi-quantitative manner, using methylation-independent PCR primers which identify genes with methylation rates over predetermined cutoffs $(10,17,27,28)$. The efficiency of the amplification of the methylated and unmethylated templates with this procedure may differ $(12,29)$, a bias that is overcome by increasing the annealing temperature (29). For DAPK and p16 genes, all samples analyzed with the MS-HRM technique turned out to be unmethylated, while the sample that appeared methylated for RASSF1A gene when analyzed with MSP, showed the same methylation status when tested by MS-HRM. The discrepancy in the obtained results between MSP and MS-HRM is probably attributable to a low prevalence of methylation in the studied sample, as MS-HRM was less sensitive than MSP and only identifies concentrations over 5\%. However, false-positive results of MSP have been reported (10), and the confirmation of MSP-positive results with another technique should be recommended. The use of MS-HRM for this confirmation in bronchial secretions needs to take into account the low proportion of methylation and 
accordingly, adjust its detection limit. An alternative option to approach this situation would be the reassessment of methylated samples by pyrosequencing (27), a sensitive and specific approach but more expensive in its performance. Then, regardless of the technique chosen for methylation analysis, analyzing sputum at least twice in independent samples and confirming positive results in bronchial secretions that appear repeatedly methylated may be recommended for the identification of true positive results.

In conclusion, although DNA methylation has been proposed as a biomarker for early detection of LC, techniques for the measure of methylation status have not been standardized, and the genes that need to be included in the analyses are not defined. Our results confirmed that sputum and BW samples are equally valid for the recognition of methylation of DAPK, p16 and RASSF1A genes in bronchial secretions. Sputum may be used as a preferential sample for the examination of methylation status as it is a sample obtained non-invasively, although the analysis of independent samples to increase the sensitivity of the test may be recommended. Due to the variability in the results according to the technique used, however, the use of combined techniques for the confirmation of positive results may increase the reliability of the methylation analysis of DAPK, p16 and RASSF1A genes.

\section{Acknowledgements}

We thank Michael Maudsley for providing an outline for this manuscript and for his support in editing and journal styling. This study was funded by Fondo de Investigación Sanitaria (http://www.isciii.es/) (PI08/1042 and PI12/02040), Fundació Parc Taulí (http://www.tauli.cat), Fundació Catalana de Pneumologia (FUCAP) (http://www.fucap.org), PII Oncologia SEPAR (http://www.separ.es/) and CIBER de Enfermedades Respiratorias - CIBERES (http://www.ciberes.org). CIBERES is an initiative of Instituto de Salud Carlos III.

\section{References}

1. Adcock IM, Tsaprouni L, Bhavsar P and Ito K: Epigenetic regulation of airway inflammation. Curr Opin Immunol 19: 694-700, 2007.

2. Barlesi F, Giaccone G, Gallegos-Ruiz MI, Loundou A, Span SW, Lefesvre P, et al: Global histone modifications predict prognosis of resected non small-cell lung cancer. J Clin Oncol 25: 4358-4364, 2007.

3. Leng S, Do K, Yingling CM, Picchi MA, Wolf HJ, Kennedy TC, et al: Defining a gene promoter methylation signature in sputum for lung cancer risk assessment. Clin Cancer Res 18: 3387-3395, 2012.

4. Malentacchi F, Forni G, Vinci S and Orlando C: Quantitative evaluation of DNA methylation by optimization of a differential-high resolution melt analysis protocol. Nucleic Acids Res 37: e86, 2009.

5. Mikeska T, Bock C, Do H and Dobrovic A: DNA methylation biomarkers in cancer: progress towards clinical implementation. Expert Rev Mol Diagn 12: 473-487, 2012.

6. Anglim PP, Alonzo TA and Laird-Offringa IA: DNA methylation-based biomarkers for early detection of non-small cel lung cancer: an update. Mol Cancer 7: 81, 2008.

7. Kim H, Kwon YM, Kim JS, Lee H, Park JH, Shim YM, et al: Tumor-specific methylation in bronchial lavage for the early detection of non-small-cell lung cancer. J Clin Oncol 22: 2363-2370, 2004.

8. Hubers AJ, van der Drift MA, Prinsen CF, Witte BI, Wang Y, Shivapurkar N, et al: Methylation analysis in spontaneous sputum for lung cancer diagnosis. Lung Cancer 84: 127-133, 2014.
9. Fahy JV, Wong H, Liu J and Boushey HA: Comparison of samples collected by sputum induction and bronchoscopy from asthmatic and healthy subjects. Am J Respir Crit Care Med 152: 53-58, 1995.

10. Kristensen LS and Hansen LL: PCR-based methods for detecting single-locus DNA methylation biomarkers in cancer diagnostics, prognostics, and response to treatment. Clin Chem 55: 1471-1483, 2009.

11. Mikeska T, Candiloro IL and Dobrovic A: The implications of heterogeneous DNA methylation for the accurate quantification of methylation. Epigenomics 2: 561-573, 2010.

12. Warnecke PM, Stirzaker C, Song J, Grunau C, Melki JR and Clark SJ: Identification and resolution of artifacts in bisulfite sequencing. Methods 27: 101-107, 2002.

13. Lorente A, Mueller W, Urdangarin E, Lazcoz P, von Deimling A and Castresana JS: Detection of methylation in promoter sequences by melting curve analysis-based semiquantitative real time PCR. BMC Cancer 8: 61, 2008.

14. Kristensen LS, Mikeska T, Krypuy M and Dobrovic A: Sensitive melting analysis after real time-methylation specific PCR (SMART-MSP): high-throughput and probe-free quantitative DNA methylation detection. Nucleic Acids Res 36: e42, 2008.

15. Kristensen LS, Wojdacz TK, Thestrup BB, Wiuf C, Hager H and Hansen LL: Quality assessment of DNA derived from up to 30 years old formalin fixed paraffin embedded (FFPE) tissue for PCR-based methylation analysis using SMART-MSP and MS-HRM. BMC Cancer 9: 453, 2009.

16. Do H, Krypuy M, Mitchell PL, Fox SB and Dobrovic A: High resolution melting analysis for rapid and sensitive EGFR and KRAS mutation detection in formalin fixed paraffin embedded biopsies. BMC Cancer 8: 142, 2008.

17. Wojdacz TK, Dobrovic A and Hansen LL: Methylation-sensitive high-resolution melting. Nat Protoc 3: 1903-1908, 2008.

18. Pin I, Gibson PG, Kolendowicz R, Girgis-Gabardo A, Denburg JA, Hargreave FE and Dolovich J: Use of induced sputum cell counts to investigate airway inflammation in asthma. Thorax 47: 25-29, 1992.

19. Pizzichini E, Pizzichini MM, Efthimiadis A, Evans S, Morris MM, Squillace D, et al: Indices of airway inflammation in induced sputum: reproducibility and validity of cell and fluid-phase measurements. Am J Respir Crit Care Med 154: 308-317, 1996.

20. Du Rand IA, Blaikley J, Booton R, Chaudhuri N, Gupta V, Khalid $\mathrm{S}$, et al: British Thoracic Society guideline for diagnostic flexible bronchoscopy in adults. Thorax 68: i1-i44, 2013.

21. Reed AP: Preparation of the patient for awake flexible fiberoptic bronchoscopy. Chest 101: 244-253, 1992.

22. Herman JG, Graff JR, Myohanen S, Nelkin BD and Baylin SB: Methylation-specific PCR: a novel PCR assay for methylation status of CpG islands. Proc Natl Acad Sci USA 93: 9821-9826, 1996.

23. Belinsky SA, Palmisano WA, Gilliland FD, Crooks LA, Divine KK, Winters SA, et al: Aberrant promoter methylation in bronchial epithelium and sputum from current and former smokers. Cancer Res 62: 2370-2377, 2002.

24. Smith E, Jones ME and Drew PA: Quantitation of DNA methylation by melt curve analysis. BMC Cancer 9: 123, 2009.

25. Belinsky SA, Liechty KC, Gentry FD, Wolf HJ, Rogers J, Vu K, et al: Promoter hypermethylation of multiple genes in sputum precedes lung cancer incidence in a high-risk cohort. Cancer Res 66: 3338-3344, 2006.

26. Belinsky SA, Grimes MJ, Casas E, Stidley CA, Franklin WA, Bocklage TJ, et al: Predicting gene promoter methylation in non-small-cell lung cancer by evaluating sputum and serum. Br J Cancer 96: 1278-1283, 2007.

27. Candiloro IL, Mikeska T and Dobrovic A: Assessing combined methylation-sensitive high resolution melting and pyrosequencing for the analysis of heterogeneous DNA methylation. Epigenetics 6: 500-507, 2011.

28. Wojdacz TK and Dobrovic A: Methylation-sensitive high resolution melting (MS-HRM): a new approach for sensitive and high-throughput assessment of methylation. Nucleic Acids Res 35: e41, 2007.

29. Shen L, Guo Y, Chen X, Ahmed S and Issa JP: Optimizing annealing temperature overcomes bias in bisulfite PCR methylation analysis. Biotechniques 42: 48-58, 2007. 